\title{
A proposal on local and adaptive determination of filter scale for edge detection
}

\author{
Domenico G. Sorrenti \\ AI and Robotics Lab., Dept. Elettronica e Informazione \\ Politecnico di Milano, Pzza Leonardo da Vinci 32, Milano, I-20133, Italy \\ fax +39223993411; email: sorrenti@elet.polimi.it
}

\begin{abstract}
In this work a proposal about local and adaptive scale selection in edge detection is presented. Such a proposal follows Canny's optimality criterion, i.e., the smallest scale that provides a minimum value for the signal to noise ratio should be selected for each edge pixel. The proposal exploits a local regularity, i.e. SNR, measure that is based on a simplified version of the Kitchen-Rosenfeld edge quality evaluator. This measure makes possible a local definition of the scale. The work has been carried out with nondirectional gaussian smoothing operators. The integration of the results, obtained at different scales on a pixel basis, into a single edge map is also tackled. Experimental work on real images is presented.
\end{abstract}

\section{Introduction}

The edge detection problem has been regarded so far as the problem of defining a filter whose convolution with the image is easily searched for the edge points, e.g., the peaks of the convoluted image. The need for multi-scale edge detection algorithms has been pointed out first by Rosenfeld and Thurston [1]. Such a need stems from admitting that images are collections of different edges, each one characterized by its own contrast and noise energy. Therefore the edge detection task turns into treating each single edge at its best, according to some optimality criterion. An important approach in the edge detection field, introducing the scale-space concept, is presented by Witkin in [2]; in this work the behavior of edges in scale-space is exploited in order to define their perceptual saliency. Basing on empirical observations Witkin defines relevant edges, i.e. to be detected, as those edges whose stability across the scales is high. On the edge positioning side of the problem, a coarse-to-fine tracking (not really well defined) try to avoid the typical errors arising in edge localization performed at large scales. The edge detection task has been clearly stated by Canny [3]. He defined criteria for the quantitative evaluation of the performance of a filter, namely detection and localization. From the criteria Canny determined a class of optimal filters for different types of edges, e.g., step edges. A specific filter in the class is defined by the scale parameter, that is the parameter defining the bandwidth of the filter, and therefore the signal to noise ratio in the convoluted image. The image is modeled as a signal corrupted by gaussian white noise. A small scale results in a nearly unaffected frequency content of the convoluted image, on the other hand a large scale attenuates the higher frequencies. A larger scale provides a larger signal to noise ratio, given the signal amplitude. A major point in Canny's work is that a trade-off between detection and localization emerged; as the scale parameter increases the detection increases, whilst localization decreases. An optimal compromise between the two criteria has to be defined; Canny proposes to select the smallest scale, provided that a minimum value for the signal to noise ratio is obtained. 
Being SNR linked to error probabilities (both marking false edges, and missing true ones), Canny's compromise means that we do not like to risk more than a certain amount. On the other hand, the selection of the minimum SNR satisfying the detection limit comes from the maximization of localization performance. In other words Canny set a priority between the criteria: first detection, then localization. In this work the take the same optimality definition.

In order to set the appropriate value for the scale parameter it is required to know the noise energy. Unfortunately it is not an easy task to locally measure the noise energy because both noise and signal affect any local measure, i.e., the signal corrupts the noise measure, as pointed out in [3]. Therefore only global measures can affordably be obtained, hence resulting in nearly single scale approaches.

Different approaches do not locally determine an optimal value for the scale parameter, but rather concentrate on the integration of results obtained at different scales. A first proposal is Canny's feature synthesis [3]; where, in a fine-to-coarse scale computation, edges not marked at smaller scales are cumulatively added to the edge map. Another relevant contribution is Bergholm's edge focusing [4] where, in a coarse-to-fine direction, edges are first detected (at the coarsest scale) and then tracked to the smallest scale. In this work integration means tracking, i.e. coarser scale is exploited to forecast edge location at the smaller scale. The algorithm generates its output at the smallest scale, therefore introducing artifacts like clouds of edge points, around the edges detected at the coarsest scale. This is a consequence of handling edges at the smallest scale of the range where edge focusing takes place, and it is unlikely that the smallest scale coincide with their optimal scale.

Differently from the above mentioned approaches, Jeong and Kim [5] aim at locally selecting the scale, on a single pixel basis. Jeong and Kim define an energy, function of the space-variant scale parameter, to be minimized under some "natural constraints". As an example of constraint they cite limiting abrupt variation of the scale parameter itself, in order to discourage fragmented edges due to noise. In the author opinion such constraint should not be wired in the algorithm whose aim is the determination of the scale parameter, but should rather emerge as the algorithm output. Moreover, in correspondence to edges between regions with different noisiness (e.g., due to different machining of the object surface) an abrupt variation of the scale parameter is desired. Jeong and Kim energy function comes from the desired behavior of the scale parameter (it should be small where intensity changes significantly and large in uniform areas), but the scale parameter generated by the algorithm is not the consequence of any optimality criterion. In particular a small error probability, i.e. a sufficiently large scale parameter, sometime is not achieved, as shown by Jeong and Kim themselves in their experimental section. Moreover, it is unclear whether a single threshold can be used to mark the edge pixels, given that the pixels are filtered at different scales.

The work here presented operates in the same mainstream as Jeong and Kim, i.e. to solve the basic conflict between detection and localization on a pixel basis. The conflict is solved according to Canny's proposal (namely, the smallest scale granting a sufficiently small error probability). Being not possible to directly measure the noise energy, a direct estimation of SNR is attempted. Integration of output at different scales is also performed in a consistent way and results are provided.

In the next section the algorithm and the local SNR estimator is presented. Experimental results and conclusions follows. 


\section{The proposed algorithm}

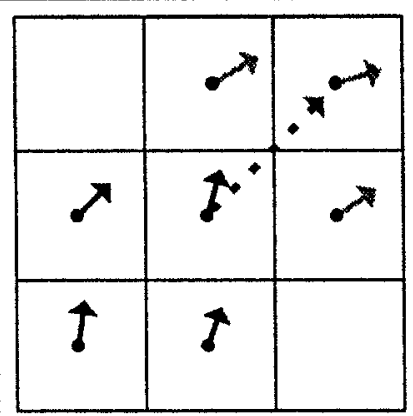

Fig. 1. Edge continuity (Left and Right) according to Kitchen and Rosenfeld; it is a function of i) central pixel edge direction (black), ii) edge direction in the neighboring pixels (right: light gray, left: dark gray), iii) the direction toward the neighbor (dotted, only the one toward the $\pi / 4$-neighbor is indicated)

In order to accomplish the above defined task we cannot follow the proved-to-fail approach of noise estimation; we rather observe that a local estimate of SNR could suffice. Such a local measure can be obtained by adapting the local edge coherence introduced by Kitchen and Rosenfeld [6]. It is briefly sketched in the following. Kitchen and Rosenfeld aim was a quantitative evaluation of the performance of different edge detectors. They defined a local measure, differently from previous approaches, and then averaged it on the whole image to give the performance figure. The local measure is built around two components, one measuring edge thinness, the second edge continuity. The two components are intuitively supposed to capture the most relevant qualities of well detected edges.

In our work edge thinness is meaningless because current edge detectors use to have an explicit thinning phase (NonMaximaSuppression), moreover we aim at an estimate of SNR, that has no impact on edge thinness. On the other hand edge continuity reveals to be strictly tied to SNR. We recall that Kitchen and Rosenfeld evaluation takes place after edge detection, when the whole edge map is available. Edge continuity rates how well formed is the edge centered on a pixel. The maximum is reached when the two neighboring pixels in the edge direction (8-conn) are edge points and their edge directions are similar to the edge direction in the central pixel (Fig. 1). On the other hand, the minimum is reached when no one of the two neighboring pixels are edge points or their edge directions are dissimilar from the edge direction in the central pixel. Kitchen and Rosenfeld expression, normalized to the $[0,1]$ interval, is computed for the three neighboring pixels in the edge direction, provided they are edge pixel. The maximum between the three value is taken as the continuity, e.g. the left continuity. Similar operations are performed for the opposite

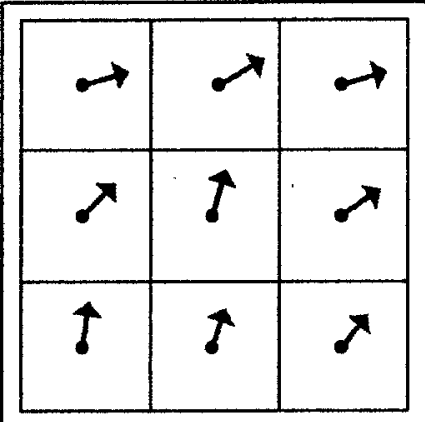

Fig. 2. SNR estimate, based on the edge direction around each pixel, central pixel direction (black), the neighbor $_{i}$ (gray); $i=0,1, \ldots, 7$ three neighbors. The average between the left and the right continuity is then taken as the local continuity figure.

We claim that, in order to give an estimate of SNR, it is enough to rely on just the continuity component of Kitchen and Rosenfeld edge evaluator, provided that the measure is monothonically increasing with SNR. Moreover, the continuity measure proposed by Kitchen and Rosenfeld can be further simplified considering the differences between the direction in the central edge and in the 8-conn neighboring pixels. In our opinion wecan build different estimators, but they can merely modify the convergence speed of the algorithm proposed in the sequel (provided they give an 
increasing output with SNR), i.e. they cannot affect its convergence. Therefore we just take, as the SNR estimate we are looking for, a very simple function of the 8 differences, namely the minimum.

\section{SNR estimate $=\min _{\mathbf{i}}\left(\right.$ CentralPixelDir.- Dir.Neighbor $\left.{ }_{\mathbf{i}}\right)$}

The SNR estimator makes possible to set up the following adaptive and multi-scale algorithm. The uniqueness of the optimum value of the scale parameter could be exploited for speeding up the process, but in the actual implementation this is not done. The scale parameter is allowed to have a finite number of values in a predetermined range. At the start the scale parameter is set for each pixel at the smallest extremum in the range; it is then increased as long as its SNR is less than the required value. Different search strategies of the best scale can only affect the speed performance of the algorithm. The algorithm resembles a trivial control system, where the user sets the desired minimum value of SNR, and the system is in charge of minimizing the difference between its desired and actual values by acting on the scale parameter.

At the end of the adaptive smoothing phase we get both the gradient module and phase.

The problem now is how to generate the final edge map. Actually this is the point where Jeong and Kim's contribution gets unclear.

Adaptive smoothing smoothes out the effects of the noise, but the module of the gradient image is still a non-thinned image; therefore a thinning phase is required. Such a computation involves comparison between adjacent

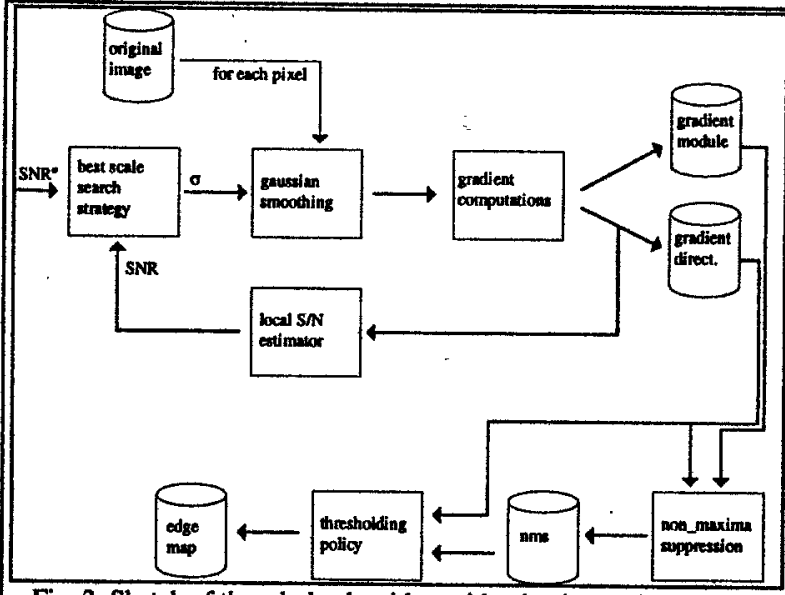

Fig. 3. Sketch of the whole algorithm with adaptive scale selection pixels (Fig. 4) that could have been filtered at different scales and therefore cannot be straightforwardly compared. Our proposal is to compare pixels at the same scale; where the central pixel, the one whose suppression is under consideration, impose its scale to the others. The central pixel has the right to be considered at its very best, i.e. at its own optimal scale. The module of the gradient of the neighboring pixels at the desired scale could be already available or not, according to the algorithm implementation. In order to avoid

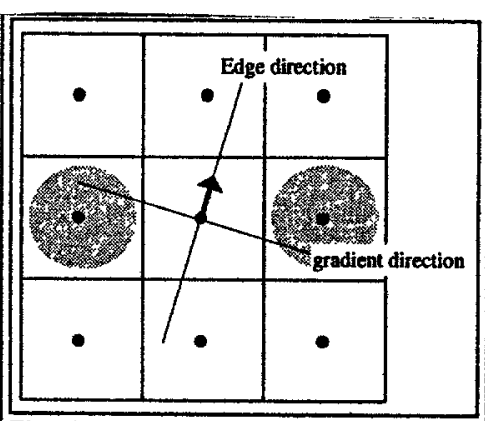

Fig. 4. In gray pixels involved in NonMaximaSuppression 
cumbersome computations and/or storage requirements, we tried to synthesize it starting from the behavior (determined off-line) of a normalized ideal step edge in the considered range of scales. We could not succeed in this approach, the attempt resulted in many kind of errors; in conclusion we propose to compute exnovo the module of the neighboring pixels whenever it is not already available. The whole edge detection algorithm therefore includes the local adaptive smoothing phase followed by a thinning phase similar to the standard NonMaximaSuppression.

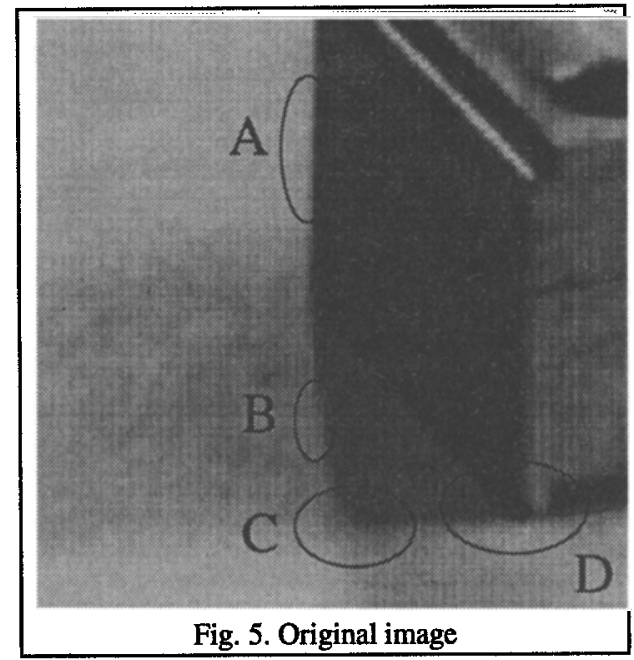

\section{Experimental results}

Relevant areas of the image used for the presentation of the experiments (Fig. 5, the object is a small piece of iron):

area $\mathrm{A}$, well contrasted edge, we expect this edge to be handled at a small scale

areas B and C, not contrasted edges; a straight

line in area B, a curve/corner in area C.

The intensity is not monothonic like it could look at first glance (see the edges detected at the smaller scales in Fig. 6).

We expect these edges to be handled at a quite large scale

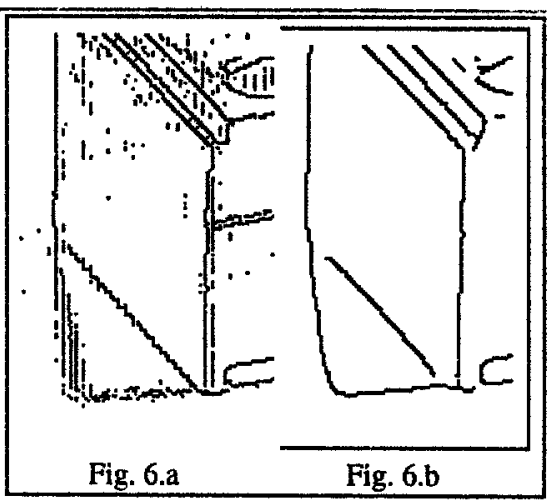
area $\mathrm{D}$, junctions of different edges

In Fig. 6.a: the edge map detected at a small scale with the single scale Canny's simple detector $(\sigma=0.3)$. $6 . \mathrm{b}$ : the same algorithm at a larger scale $(\sigma=2.5)$. 6.c: the edge map detected by the proposed algorithm ( $\sigma$ is in the range $[0.3,2.5])$. Fig. 7. shows the output of Canny's single scale superimposed to the output of the proposed algorithm. Light gray: single scale, dark gray: multi-scale, black: pixel edges detected by both the algorithms. The mislocalization and distortion inherent in the output of the single scale are magnified by using a scale $(\sigma=5)$ higher than the maximum in the range of the multi-scale $(\sigma=2.5)$ in order to ease observation of mislocalization errors. In area $A$ most edges are handled at $\sigma \equiv 0.7$; in

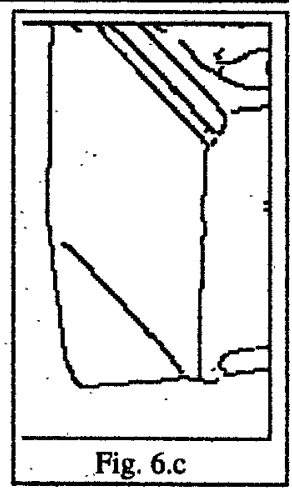
areas $B$ and $C$ : most edges are handled at $\sigma=2.5$; in area $C$ a few would require to be handled at a scale larger than the largest in the range (they did not reach the SNR threshold); in area D: most edges are handled at relatively small scales (with the exception of those on the shadow edge) 


\section{Conclusions}

A local and adaptive scale selection algorithm for edge detection is presented. It aims at the selection of the best scale for each pixel, where best is defined as in [3] as the smallest scale that provides a minimum value for the signal to noise ratio. The algorithm exploits a local regularity, i.e. SNR, measure that is based on a simplified version of the Kitchen-Rosenfeld edge quality evaluator. This makes possible a definition of the scale on a basis as local as the measure support. The results, obtained at different scales for each pixel, are integrated into a single edge map by means of a NonMaximaSuppression procedure similar the standard one. Experimental work confirms that the approach provides a good answer to the local and adaptive edge detection problem. The limits of the algorithm, in the author opinion, are a consequence of the fact that adaptive smoothing is performed with a standard non-directional smoothing filter; therefore, where the signal amplitude is small compared to the noise and the edge

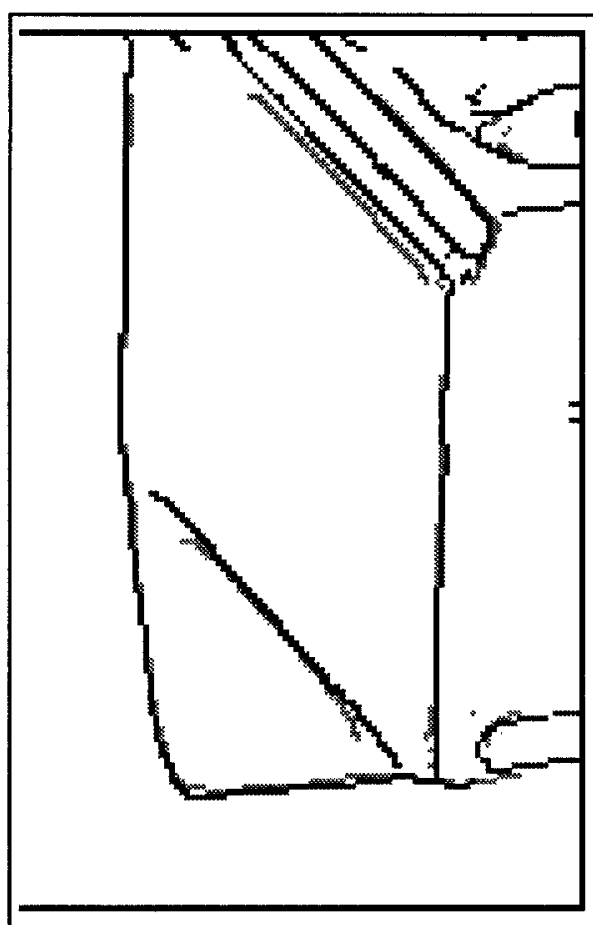

Fig. 7. Comparison of edges detected by a (large) fixed scale and by the proposed multiscale algorithm.

is short interaction with neighboring edges arises. Integration of a discontinuitypreserving approach like [7] is under investigation, as well as recursive implementation of linear filtering [8]. The algorithm is well suited for parallel implementation on loosely-coupled MIMD machines because it involves highly local computations.

\section{References}

[1] Rosenfeld A., Thurston M., "Edge and curve detection for visual scene analysis", IEEE Trans. on Computers, May 1971

[2] Witkin A. P., "Scale-space filtering", Proc. Int. Joint Conf. Artificial Intelligence 1983

[3] Canny J., "A computational approach to edge detection", IEEE Trans. on PAMI, November 1986

[4] Bergholm F., "Edge focusing", IEEE Trans. on PAMI November 1987

[5] Jeong H., Kim C. I., "Adaptive determination of filter scale for edge detection", IEEE Trans. on PAMI, May 1992

[6] Kitchen L., Rosenfeld A., "Edge evaluation using local edge coherence", IEEE Trans. on SMC, September 1981

[7] Perona P., Malik J., "Scale-space and edge detection using anisotropic diffusion", IEEE Trans. on PAMI, July 1990

[8] Deriche R., "Fast algorithms for low-level vision", IEEE Trans. on PAMI, January 1990 